\title{
SVM-Based Selection of Colour Space Experts for Face Authentication
}

\author{
Mohammad T. Sadeghi ${ }^{1}$, Samaneh Khoshrou ${ }^{1}$, and Josef Kittler ${ }^{2}$ \\ ${ }^{1}$ Signal Processing Research Lab., Department of Electronics \\ University of Yazd, Yazd, Iran \\ ${ }^{2}$ Centre for Vision, Speech and Signal Processing \\ School of Electronics and Physical Sciences \\ University of Surrey, Guildford GU2 7XH, UK \\ \{M.Sadeghi, J.Kittler\}@surrey.ac.uk
}

\begin{abstract}
We consider the problem of fusing colour information to enhance the performance of a face authentication system. The discriminatory information potential of a vast range of colour spaces is investigated. The verification process is based on the normalised correlation in an LDA feature space. A sequential search approach which is in principle similar to the "plus L and take away R" algorithm is applied in order to find an optimum subset of the colour spaces. The colour based classifiers are combined using the SVM classifier. We show that by fusing colour information using the proposed method, the resulting decision making scheme considerably outperforms the intensity based verification system.
\end{abstract}

\section{Introduction}

The spectral property of the skin albedo is known to provide useful biometric information for face recognition. Measured in terms of colour, it has been exploited directly in terms of features derived from the colour histogram [7]. Such features are then used to augment the face feature space in which the matching of a probe image against a template is carried out. Alternatively, the spectral information can be used indirectly, by treating each R, G, B colour channel as a separate image. The face matching scores computed for the respective channels are then combined to reach the final decision about the probe image identity. This indirect approach has the advantage that an existing face recognition system can simply be applied to create the respective colour space experts, without any structural redesign. This indirect approach has been investigated extensively in [13 6]. In these studies the colour face experts were designed in different colour spaces, such as R, G, B, the intensity image, normalised green and an opponent colour channel, or colour channels decorrelated by the Principal Component Analysis (PCA). The merits of the information conveyed by the various channels individually, as well as their combined effect, have been evaluated. It has been demonstrated that some of the colour spaces provide more powerful representation of the face skin properties than others, offering significant improvements in performance as compared to the raw intensity image. In 
this paper we extend the work to other colour representations suggested in the literature. In total, 15 different colour spaces are considered, giving rise to 45 different colour channels. Assuming that face experts based on all these channels are available, it is pertinent to ask which channels provide complementary information and how the expert scores should be fused to achieve the best possible performance of the face recognition system. In 12 the fusion problem was solved by selecting the best expert or a group of experts dynamically with the help of a gating function learnt for each channel. In the present study we formulate the colour expert fusion problem as a feature selection problem. The colour spaces for fusion are selected using a sequential search approach similar to the Plus L and Take Away $\mathrm{R}$ algorithm.

An important contributing factor in the selection algorithm is the "fusion rule" used for combining the colour based classifiers. In 11 untrained methods such as averaging and voting schemes were used for this purpose. However, in different applications it has been demonstrated that trained approaches such as Support Vector Machines (SVMs) have the potential to outperform the simple fusion rules, especially when a large enough training data is available. The main aim of this paper is to study the performance of the proposed colour selection algorithm using the SVM classifiers. Surprisingly good results are obtained using the proposed method.

The paper is organised as follows. In the next section different colour spaces adopted in different machine vision applications are reviewed. The face verification process is briefly discussed in Section 3 . The proposed method of colour space selection is described in Section 4. The experimental set up is detailed in Section 5. Section 6 presents the results of the experiments. Finally, in Section 7 the paper is drawn to conclusion.

\section{Colour Spaces}

For computer displays, it is most common to describe colour as a set of three primary colours: Red, Green and Blue. However, it has been demonstrated that in different applications using different colour spaces could be beneficial. In this section some of the most important colour spaces are reviewed. Considering the $R, G, B$ system as the primary colour space, we can classify the other colour spaces into two main categories: Linear and Nonlinear transformation of the $R$, $G, B$ values.

\subsection{Linear Combination of $R, G, B$}

$C M Y$-based colour space is commonly used in colour printing systems. The name $C M Y$ refers to cyan, magenta and yellow. The $R G B$ values can be converted to $C M Y$ values using:

$$
C=255-R, \quad M=255-G, \quad Y=255-B
$$

There are several CIE-based colour spaces,but all are derived from the fundamental $X Y Z$ space [2]. A number of different colour spaces including $Y U V$, 
$Y I Q, Y E S$ and $Y C_{b} C_{r}$ are based on separating luminance from chrominance (lightness from colour). These spaces are useful in compression and other image processing applications. Their formal definition can be found in [2].

$I 1 I 2 I 3$ or Ohta's features [8] were first introduced for segmentation as optimised colour features and are shown in equations:

$$
I 1=\frac{R+G+B}{3.0}, \quad I 2=R-B, \quad I 3=2 G-R-B
$$

LEF Colour Space defines a colour model that combines the additivity of the RGB model with the intuitiveness of the hue-saturation-luminance models by applying a linear transformation to the $R G B$ cube.

\subsection{Nonlinear Combination of $R, G, B$}

The chromaticities for the normalised $R G B$ are obtained by normalising the $R G B$ values with the intensity value, $I$ :

$$
r=R / I, \quad g=G / I, \quad b=B / I
$$

where $I=(R+G+B) / 3$. Similar equations are used for normalising the $X Y Z$ values. The result is a $2 \mathrm{D}$ space known as the CIE chromaticity diagram. The opponent chromaticity space is also defined as

$$
r g=r-g, \quad y b=r+g-2 b
$$

Kawato and Ohya [5] have used the $a b$ space which is derived from NCC rgchromaticities as:

$$
a=r+g / 2, \quad b=\sqrt{3} /(2 g)
$$

In [16, two colour spaces namely $P 1$ and $P 2$ have been defined by circulating the $r, g$ and $b$ values in equation 4, Log-opponent (or Log-opponent chromaticity) space has been applied to image indexing in [1]. The space is presented by equations:

$$
\begin{aligned}
& L n_{r g}=\ln (R / G)=\ln R-\ln G \\
& L n y b=\ln \left(\frac{R \cdot G}{B^{2}}\right)=\ln R+\ln G-2 \ln B
\end{aligned}
$$

TSL (Tint - Saturation - Lightness) colour space is also derived from NCC rg-chromaticities.

$l 1 l 2 l 3$ colour space as presented in [4 has been adopted for colour-based object recognition. Many people find $H S$-spaces $(H S V, H S B, H S I, H S L)$ intuitive for colour definition. For more information about the relevant equations used in this study, the reader is referred to [3]. 


\section{Face Verification Process}

The face verification process consists of three main stages: face image acquisition, feature extraction, and finally decision making. The first stage involves sensing and image preprocessing the result of which is a geometrically registered and photometrically normalised face image. Briefly, the output of a physical sensor (camera) is analysed by a face detector and once a face instance is detected, the position of the eyes is determined. This information allows the face part of the image to be extracted at a given aspect ratio and resampled to a pre-specified resolution. The extracted face image is finally photometrically normalised to compensate for illumination changes.

The raw colour camera channel outputs, $R, G$ and $B$ are converted according to the desired image representation spaces. In this study different colour spaces reviewed in the previous section were considered.

In the second stage of the face verification process the face image data is projected into a feature space. The final stage of the face verification process involves matching and decision making. Basically the features extracted for a face image to be verified, $\mathbf{x}$, are compared with a stored template, $\boldsymbol{\mu}_{i}$, that was acquired on enrolment. In [14], it was demonstrated that the Gradient Direction (GD) metric or Normalised Correlation (NC) function in the Linear Discriminant Analysis (LDA) feature space works effectively in the face verification systems. In this study we adopted the NC measure in the LDA space. The score, $s$, output by the matching process is then used for decision making. In our previous studies [1] 6], global or client specific thresholding method were used for decision making. In this work, the above mentioned thresholding methods are compared to decision making using the Support Vector Machines.

If the score computation is applied to different colour spaces separately, we end up with a number of scores, $s_{k}=s\left(\mathbf{x}_{k}\right), k=1,2, \ldots, N$ which then have to be fused to obtain the final decision. The adopted fusion method is studied in the next section.

\section{Colour Space Selection}

One of the most exciting research directions in the field of pattern recognition and computer vision is classifier fusion. Multiple expert fusion aims to make use of many different designs to improve the classification performance. The approach we adopted for selecting the best colour space(s) is similar in principal to the sequential feature selection methods in pattern recognition [10. In this study, the Sequential Forward Selection (SFS), Sequential Backward Selection (SBS) and Plus'L' and Take away 'R' algorithms were examined for selecting an optimum subset of the colour spaces. Two untrained fusion rules, the sum rule and the voting scheme and a trained fusion method, the Support Vector Machines are used in order to combine the scores of the colour based classifiers. The selection procedure keeps adding or taking away features (colour spaces in our case) until the best evaluation performance is achieved. The selected colour spaces are then used in the test stage. 


\subsection{Support Vector Machines}

A Support Vector Machine is a two-class classifier showing superior performance to other methods in terms of Structural Risk Minimisation 15. For a given training sample $\left\{\mathbf{x}_{i}, y_{i}\right\}, i=1, \ldots, N$, where $\mathbf{x}_{i} \in R^{D}$ is the object marked with a label $y_{i} \in\{-1,1\}$, it is necessary to find the direction $\mathbf{w}$ along which the margin between objects of two classes is maximal. Once this direction is found the decision function is determined by threshold $b$ :

$$
y(\mathbf{x})=\operatorname{sgn}(\mathbf{w} \cdot \mathbf{x}+b)
$$

The threshold is usually chosen to provide equal distance to the closest objects of the two classes from the discriminant hyperplane $\mathbf{w} \cdot \mathbf{x}+b=0$, which is called the optimal hyperplane. When the classes are linearly non-separable some objects can be shifted by a value $\delta_{i}$ towards the right class. This converts the original problem into one which exhibits linear separation. The parameters of the optimal hyperplane and the optimal shifts can be found by solving the following quadratic programming problem:

$$
\begin{aligned}
& \text { minimise } \mathbf{w} \cdot \mathbf{w}+\mathcal{C} \sum_{i=1}^{N} \delta_{i} \\
& \text { subject to: } \\
& y_{i}\left(\mathbf{w} \cdot \mathbf{x}_{i}+b\right) \geq 1-\delta_{i}, \quad \delta_{i} \geq 0 \quad i=1, \ldots, N
\end{aligned}
$$

where parameter $\mathcal{C}$ defines the penalty for shifting the objects that would otherwise be misclassified in the case of linearly non separable classes.

The QP problem is usually solved in a dual formulation

$$
\begin{aligned}
& \text { minimise } \sum_{i=1}^{N} \alpha_{i}-\frac{1}{2} \sum_{i=1}^{N} \sum_{j=1}^{N} \alpha_{i} \alpha_{j} y_{i} y_{j} \mathbf{x}_{i} \cdot \mathbf{x}_{j} \\
& \text { subject to: } \\
& \sum_{i=1}^{N} \alpha_{i} y_{i}=0, \quad 0 \leq \alpha_{i} \leq C \quad i=1, \ldots, N
\end{aligned}
$$

Those training objects $\mathbf{x}_{i}$ with $\alpha_{i}>0$ are called Support Vectors, because only they determine direction $\mathbf{w}$ :

$$
\mathbf{w}=\sum_{i=1, \alpha_{i}>0}^{N} \alpha_{i} y_{i} \mathbf{x}_{i}
$$

The dual QP problem can be rapidly solved by the Sequential Minimal Optimisation method, proposed by Platt [9]. This method exploits the presence of linear constraints in (9). The QP problem is iteratively decomposed into a series of one variable optimisation problems which can be solved analytically.

For the face verification problem, the size of the training set for clients is usually less than the one for impostors. In such a case, the class of impostors is represented better. Therefore, it is necessary to shift the optimal hyperplane 
towards the better represented class. In this work, the size of the shift is determined in the evaluation step considering the Equal Error Rate criterion.

\section{Experimental Design}

The aim of the experiments is to show that by fusing the sensory data used by component experts, the performance of the multiple classifier system improves considerably. We use the XM2VTS database 1 and its associated experimental protocols for this purpose.

The XM2VTS database is a multi-modal database consisting of face images, video sequences and speech recordings taken of 295 subjects at one month intervals. Since the data acquisition was distributed over a long period of time, significant variability of appearance of clients, e.g. changes of hair style, facial hair, shape and presence or absence of glasses, is present in the recordings.

For the task of personal verification, a standard protocol for performance assessment has been defined. The so called Lausanne protocol splits randomly all subjects into a client and impostor groups. The client group contains 200 subjects, the impostor group is divided into 25 evaluation impostors and 70 test impostors. The XM2VTS database contains 4 sessions. Eight images from 4 sessions are used.

From these sets consisting of face images, training set, evaluation set and test set are built. There exist two configurations that differ by a selection of particular shots of people into the training, evaluation and test sets. The training set is used to construct client models. The evaluation set is selected to produce client and impostor access scores, which are used for designing the required classifier. The score classification is done either by the SVM classifier or by thresholding. The thresholds are set either globally $(G T)$ or using the client specific thresholding (CST)technique [6]. According to the Lausanne protocol the threshold is set to satisfy the Equal Error Rate criterion, i.e. the operating point where the false rejection rate (FRR) is equal to the false acceptance rate (FAR). False acceptance is the case where an impostor, claiming the identity of a client, is accepted. False rejection is the case where a client, claiming his true identity, is rejected. The evaluation set is also used in fusion experiments (classifier combination) for training. The SVM-based sequential search algorithms pick the best colour spaces using this set of data.

Finally the test set is selected to simulate realistic authentication tests where impostor's identity is unknown to the system. The performance measures of a verification system are the False Acceptance rate and the False Rejection rate.

The original resolution of the image data is $720 \times 576$. The experiments were performed with a relatively low resolution face images, namely $64 \times 49$. The results reported in this article have been obtained by applying a geometric face registration based on manually annotated eyes positions. Histogram equalisation was used to normalise the registered face photometrically.

${ }^{1}$ http://www.ee.surrey.ac.uk/Research/VSSP/xm2vtsdb/ 


\section{$6 \quad$ Experimental Results}

Table \shows the performance of the face verification system using the individual colour spaces considering the first configuration of the Lausanne protocol. The decision boundary in the NC space was determined using the SVMs. The values in the table indicate the FAR and FRR in both evaluation and test stages. As we expect, the best performance is obtained neither in the original RGB spaces nor in the intensity space. Some other colour spaces such as U in the YUV space or opponent chromaticities individually can lead to better results. Table 2 shows some results of the same experiments considering the second XM2VTS protocol configuration.

As mentioned earlier, in similar experiments the global or client specific thresholding techniques were used for decision making. Figure 1 contains plots of the total error rate in different colour spaces in the evaluation and test stages. These results demonstrate that although in most of the cases, the SVMs work better than the GT technique, but the CST leads to better or comparable results.

Table 1. Identity verification results using different colour spaces. Classification boundary was determined using the SVMs.(configuration 1).

\begin{tabular}{|c||c|c|c|c|c|c|c|c|c|}
\hline subspace & $\mathrm{R}$ & $\mathrm{G}$ & $\mathrm{B}$ & $\mathrm{I}$ & $\mathrm{H}$ & Sat & Val & $\mathrm{r}$ & $\mathrm{g}$ \\
\hline FAR Eval & 2.19 & 1.97 & 1.75 & 2.14 & 1.92 & 1.76 & 2.19 & 1.93 & 1.48 \\
\hline FRR Eval. & 2.17 & 2 & 1.83 & 2.17 & 1.83 & 1.66 & 2.16 & 1.83 & 1.5 \\
\hline FAR Test & 2.41 & 1.97 & 1.97 & 2.17 & 1.84 & 1.81 & 2.46 & 2.08 & 1.47 \\
\hline FRR Test & 1.5 & 1.75 & 1.75 & 1.25 & 0.5 & 1.25 & 2 & 0.75 & 1 \\
\hline \hline subspace & $\mathrm{b}$ & $\mathrm{T}(\mathrm{TSL})$ & $\mathrm{S}(\mathrm{TSL})$ & $\mathrm{L}(\mathrm{TSL})$ & $\mathrm{V}(\mathrm{YUV})$ & $\mathrm{rg}$ & $\mathrm{U}(\mathrm{YUV})$ & $\mathrm{Cr}$ & $\mathrm{I} 2$ \\
\hline FAR Eval. & 1.69 & 1.52 & 1.31 & 2.11 & 2.31 & 1.49 & 1.91 & 1.82 & 2.31 \\
\hline FRR Eval & 1.66 & 1.5 & 1.33 & 2.166 & 2.33 & 1.5 & 1.83 & 1.83 & 2.33 \\
\hline FAR Test & 1.73 & 1.32 & 1.53 & 2.1491 & 2.26 & 1.60 & 1.75 & 2.21 & 2.3 \\
\hline FRR Test & 1.25 & 1 & 1.75 & 1.5 & 0.75 & 1.25 & 0 & 1.5 & 0.75 \\
\hline \hline subspace & $\mathrm{I} 3$ & $\mathrm{E}(\mathrm{LEF})$ & $\mathrm{F}(\mathrm{LEF})$ & $\mathrm{X}(\mathrm{CIE})$ & $\mathrm{Y}(\mathrm{CIE})$ & $\mathrm{Z}(\mathrm{CIE})$ & $\mathrm{Y}(\mathrm{YES})$ & $\mathrm{E}(\mathrm{YES})$ & $\mathrm{S}(\mathrm{YES})$ \\
\hline FAR Eval. & 1.67 & 2.1 & 1.66 & 2.27 & 2.11 & 2 & 2.05 & 1.82 & 2 \\
\hline FRR Eval. & 1.6 & 2.166 & 1.67 & 2.33 & 2.166 & 2 & 2 & 1.83 & 2 \\
\hline FAR Test & 1.69 & 2.21 & 1.60 & 2.38 & 2.14 & 2.25 & 2.10 & 1.65 & 1.82 \\
\hline FRR Test & 0.75 & 0.5 & 0.5 & 1.25 & 1.5 & 1.75 & 1.5 & 0.75 & 0.25 \\
\hline \hline subspace & $\mathrm{I}(\mathrm{YIQ})$ & $\mathrm{Q}(\mathrm{YIQ})$ & $\mathrm{a}(\mathrm{ab})$ & $\mathrm{b}(\mathrm{ab})$ & $\mathrm{Lnrg}$ & $\mathrm{Lnyb}$ & 11 & 12 & 13 \\
\hline FAR Eval. & 2.17 & 1.85 & 1.79 & 1.52 & 1.22 & 1.49 & 2.05 & 2.35 & 1.71 \\
\hline FRR Eval. & 2.16 & 1.83 & 1.83 & 1.5 & 1.16 & 1.5 & 2 & 2.33 & 1.67 \\
\hline FAR Test & 2.48 & 1.75 & 1.92 & 1.58 & 1.35 & 1.59 & 1.74 & 2.46 & 1.58 \\
\hline FRR Test & 1.5 & 0.75 & 1.25 & 1.2500 & 1.75 & 1.25 & 1 & 1.25 & 1.50 \\
\hline \hline subspace & LHSL $)$ & $\mathrm{Xn}$ & $\mathrm{Yn}$ & $\mathrm{Zn}$ & $\mathrm{C}(\mathrm{CMY})$ & $\mathrm{M}(\mathrm{CMY})$ & $\mathrm{Y}(\mathrm{CMY})$ & $\mathrm{bg}$ & \\
\hline FAR Eval. & 2.33 & 1.80 & 1.65 & 1.52 & 2.47 & 2.02 & 1.74 & 1.66 & \\
\hline FRR Eval. & 2.33 & 1.83 & 1.66 & 1.5 & 2.5 & 2 & 1.83 & 1.66 & \\
\hline FAR Test & 2.42 & 1.90 & 1.69 & 1.57 & 2.79 & 2.02 & 1.97 & 1.73 & \\
\hline FRR Test & 1 & 1 & 1.25 & 0.5 & 1.75 & 1.75 & 1.75 & 0.75 & \\
\hline
\end{tabular}


Table 2. Identity verification results using some of the colour spaces (configuration 2)

\begin{tabular}{|c||c|c|c|c|c|c|c|c|c|c|}
\hline subspace & $\mathrm{R}$ & $\mathrm{G}$ & $\mathrm{B}$ & $\mathrm{I}$ & $\mathrm{H}$ & $\mathrm{S}$ & $\mathrm{V}$ & $\mathrm{r}$ & $\mathrm{g}$ & $\mathrm{b}$ \\
\hline FAR Eval. & 1.26 & 1.25 & 1.25 & 1.24 & 1.25 & 1.23 & 1.25 & 1.36 & 0.74 & 1.34 \\
\hline FRR Eval. & 1.25 & 1.25 & 1.25 & 1.25 & 1.25 & 1.25 & 1.25 & 1.25 & 0.75 & 1.25 \\
\hline FAR Test & 1.65 & 1.67 & 1.87 & 1.80 & 1.14 & 1.71 & 1.61 & 2.02 & 0.76 & 1.67 \\
\hline FRR Test & 1.5 & 1.5 & 1.5 & 1.5 & 0.5 & 1 & 1.5 & 1.5 & 0.5 & 0.75 \\
\hline
\end{tabular}

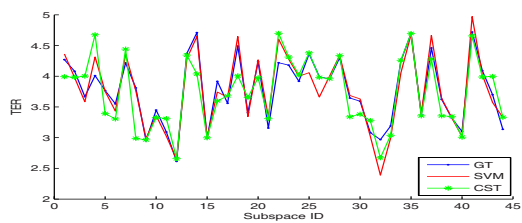

(a) Config. 1, Evaluation

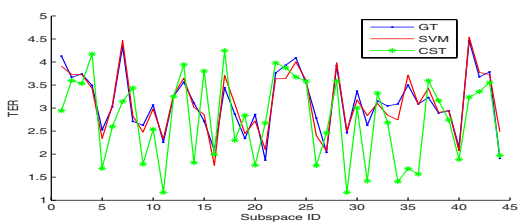

(c) Config. 1, Test

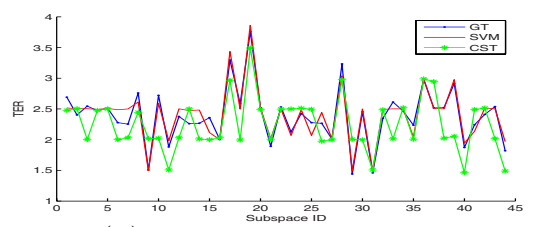

(b) Config. 2, Evaluation

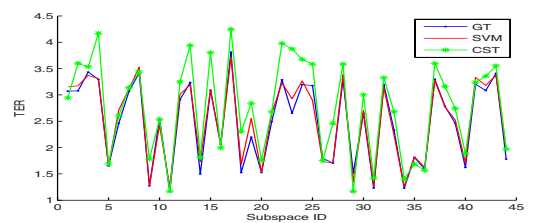

(d) Config. 2, Test

Fig. 1. Verification results in different colour spaces. The decision boundary has been determined using GT, SVMs or CST.

In the next step, the adopted search method, Plus ' $L$ ' and Take away 'R' algorithm was used for selecting a subset of colour spaces. Figure 2 shows the resulting error rates for different number of colour spaces in Configurations 1 and 2. In the search algorithm $L=2$ and $R=1$. Before fusing, the scores associated to each colour space were appropriately normalised. The normalised scores were then considered as a feature vector. The SVM classifier was finally used for decision making.

Table 3 contains the fusion results obtained using the adopted "Plus 2 and take away 1" algorithm for Configurations 1 and 2. These results were obtained by score fusion using the averaging rule or the SVMs. Note that using the search algorithm, colour spaces are selected from the evaluation data for the whole data set. However, the algorithm is flexible so that, for different conditions, different spaces can be adopted adaptively. In the case of the experimental protocols of the XM2VTS database using the SVM classifier Lnrg, a(ab), V(HSV) spaces have been selected for the first configuration while b(ab),U(YUV), bg(opp-chroma), $\mathrm{Cr}, \mathrm{X}(\mathrm{CIE}), \mathrm{I}, \mathrm{V}(\mathrm{HSV}), \mathrm{H}(\mathrm{HSV})$ and $\mathrm{S}(\mathrm{YES})$ have been adopted for the second one. These results first of all demonstrate that the proposed colour selection algorithm considerably improves the performance of the face verification system. Moreover, they also show that although not much gain in performance can be 


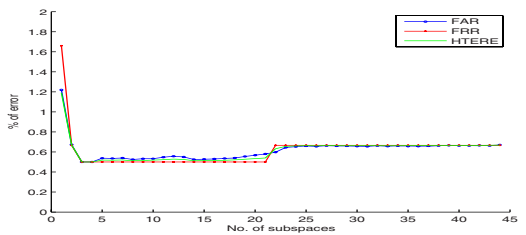

(a) Config. 1, Evaluation

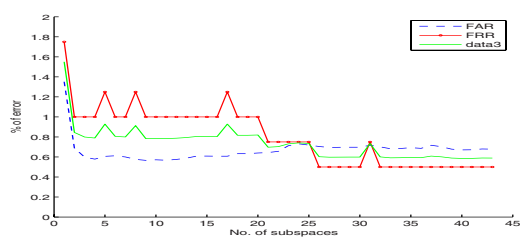

(c) Config. 1, Test

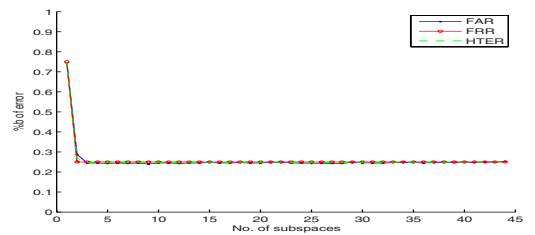

(b) Config. 2, Evaluation

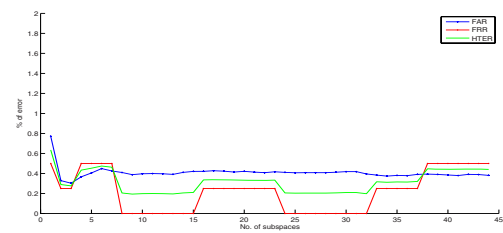

(d) Config. 2, Test

Fig. 2. SVM-based Plus 2 and Take away 1 results

Table 3. Verification results using the proposed colour selection and fusion methods

\begin{tabular}{|c||c||c|c|c||c|c|c|}
\hline \multirow{2}{*}{ Configuration } & \multirow{2}{*}{ Fusion rule } & \multicolumn{3}{c||}{ Evaluation } & \multicolumn{3}{c|}{ Test } \\
\cline { 2 - 8 } & & FAR & FRR & TER & FAR & FRR & TER \\
\hline \multirow{2}{*}{1} & Averaging & 0.48 & 0.5 & 0.98 & 0.55 & 1 & 1.55 \\
\cline { 2 - 8 } & SVMs & 0.5 & 0.5 & 1 & 0.59 & 1 & 1.59 \\
\hline \multirow{2}{*}{2} & Averaging & 0.19 & 0.25 & 0.44 & 0.27 & 0.25 & 0.52 \\
\cline { 2 - 7 } & SVMs & 0.24 & 0.25 & 0.49 & 0.38 & 0 & 0.38 \\
\hline
\end{tabular}

obtained from SVMs in the colour spaces individually (figure 1), combining the colour based classifiers using SVMs would overall be beneficial.

\section{Conclusions}

We addressed the problem of fusing colour information for face authentication. In a face verification system which is based on the normalised correlation measure in the LDA face space, an SVM-based sequential search approach similar to the "plus $\mathrm{L}$, and take away R" algorithm was applied in order to find an optimum subset of the colour spaces. Using the proposed method, the performance of the verification system was considerably improved as compared to intensity only or the other colour spaces. Within the framework of the proposed method, we showed that fusing colour based classifiers using SVMs outperforms the simple averaging rule.

Acknowledgements. The financial support from Iran Telecommunication Research Centre is gratefully acknowledged. A partial support from the EU Network of Excellence Biosecure and from the EPSRC Grant GR/S98528/01 is also gratefully acknowledged. 


\section{References}

1. Berens, J., Finlayson, G.: Log-opponent chromaticity coding of colour space. In: Proceedings of the Fourth IEEE International Conference on Pattern Recognition, pp. 1206-1211. IEEE Computer Society Press, Los Alamitos (2000)

2. Colantoni, P., et al.: Color space transformations. Technical report, http://www.raduga-ryazan.ru/files/doc/colorspacetransform95.pdf

3. Foley, J., van Dam, A., Feiner, S., Hughes, J.: Computer graphics: principles and practice, 2nd edn. Addison-Wesley Longman Publishing Co. Inc., Boston, MA, USA (1996)

4. Gevers, T., Smeulders, A.: Colour based object recognition. In: ICIAP, vol. 1, pp. 319-326 (1997)

5. Kawato, S., Ohya, J.: Real-time detection of nodding and head-shaking by directly detecting and tracking the "between-eyes". In: Proceedings of the Fourth IEEE International Conference on Automatic Face and Gesture Recognition, pp. 40-45. IEEE Computer Society Press, Los Alamitos (2000)

6. Kittler, J., Sadeghi, M.: Physics-based decorrelation of image data for decision level fusion in face verification. In: Roli, F., Kittler, J., Windeatt, T. (eds.) MCS 2004. LNCS, vol. 3077, pp. 354-363. Springer, Heidelberg (2004)

7. Marcel, S., Bengio, S.: Improving face verification using skin colour information. In: 16th International Conference on Pattern Recognition, vol. 2, pp. 20378-20382 (2002)

8. Ohta, Y., Kanade, T., Sakai, T.: Colour information for region segmentation. Computer Graphics and Image Processing 13(3), 222-241 (1980)

9. Platt, J.: Sequential minimal optimization: A fast algorithm for training support vector machines. Technical Report 98-14, Microsoft Research, Redmond, Washington (April 1998)

10. Pudil, P., Novovicova, J., Kittler, J.: Floating search methods in feature selection. Pattern Recognition Letters 15, 1119-1125 (1994)

11. Sadeghi, M., Khoshrou, S., Kittler, J.: Colour feature selection for face authentication. In: Proceedings of the International Conference on Macine Vision Applications, MVA'07, Japan, May 2007 (2007)

12. Sadeghi, M., Khoshrou, S., Kittler, J.: Confidence based gating of colour features for face authentication. In: Proceedings of the 7th International Workshop on Multiple Classifier System, MCS'07, Czech Republi, May 2007, pp. 121-130 (2007)

13. Sadeghi, M., Kittler, J.: A comparative study of data fusion strategies in face verification. In: The 12th European Signal Processing Conference, Vienna, Austria, 6-10 September 2004 (2004)

14. Sadeghi, M., Kittler, J.: Decision making in the LDA space: Generalised gradient direction metric. In: The 6th International Conference on Automatic Face and Gesture Recognition, Seoul, Korea, May 2004, pp. 248-253 (2004)

15. Vapnik, V.: The Nature of Statistical Learning Theory. Springer, New York (1995)

16. Vertan, C., Cuic, M., Boujemaa, N.: On the introduction of a chrominance spectrum and its applications. In: Proceedings of the First International Conference on Colour in Graphics and Image Processing, 1-4 October 2000, pp. 214-218 (2000) 